\title{
Análisis de la Administración Financiera en el Sector Público
}

\author{
Ramírez Casco, Andrea del Pilar * \\ https://orcid.org/0000-0002-1128-2272 \\ Calderón Moran, Edison Vinicio* \\ https://orcid.org/0000-0002-4016-6801 \\ *Escuela Superior Politécnica de Chimborazo \\ E-mail: andrea.ramirez@espoch.edu.ec
}

Recibido: 24 de noviembre de 2020

Aprobado: 22 de diciembre de 2020

\section{Resumen}

La presente investigación se basa en realizar un análisis de la Administración Financiera en el sector público, llegando a considerar una medición a través del uso de los presupuestos y el cumplimiento de sus competencias con eficacia, con el fin de resaltar la importancia que tiene el uso como potencial informativo a la hora de examinar la situación financiera de las entidades públicas, para poder evaluar su cumplimiento con relación al servicio que presta a la ciudadanía, los resultados de este trabajo brindarán a la sociedad una información sistematizada de la realidad financiera de las entidades púbicas, la cual será de gran importancia para la toma de decisiones por parte de los distintos sujetos interesados en el funcionamiento de las organizaciones y mejoras en la gestión financiera, así como también servirá de guía para que el sector público puedan tener una información financiera que permita comparar con otras de su segmento por la actividad que desarrolla. Finalmente, para analizar la Administración Financiera de cada entidad, este estudio compilará indicadores que midan el desempeño y los resultados de su gestión, la cual permite tener una visión integral de la situación en la que se encuentran estas, y el servicio que dan a la comunidad.

Palabras clave: Administración Financiera, Presupuesto, Empresas. 


\title{
Analysis of Financial Administration in the Public Sector
}

\begin{abstract}
This research is based on carrying out an analysis of Financial Administration in the public sector, coming to consider a measurement through the use of budgets and the fulfillment of its competencies effectively, in order to highlight the importance of the use of As an informative potential when examining the financial situation of public entities, in order to evaluate their compliance in relation to the service provided to citizens, the results of this work will provide society with systematized information on the financial reality of the entities which will be of great importance for decision-making by the different subjects interested in the operation of organizations and improvements in financial management, as well as serve as a guide so that the public sector can have financial information that allows you to compare with others in its segment by the activity it develops. Finally, to analyze the Financial Administration of each entity, this study will compile indicators that measure the performance and results of their management, which allows a comprehensive view of the situation in which they are, and the service they provide to the company. community.
\end{abstract}

Keywords: Financial Management, Budget, Companies.

\section{Introducción}

Las instituciones que conforman el sector público, tienen el deber de satisfacer las demandas de la ciudadanía para lo cual necesitan contar con una adecuada Administración Financiera ya que su estructura presupuestaria de ingresos y gastos les permita la sostenibilidad de los diferentes proyectos de inversión que realizan para atender la demanda creciente de bienes y servicios públicos, la cual será la encargada de proveer a los ciudadanos para que mejoren las condiciones de vida de la sociedad.

Se hace necesario identificar las características de la Administración Financiera, como también las diferentes normativas que rigen a las instituciones del sector público teniendo en cuenta que las mismas deben realizar el ciclo presupuestario como premisa de cumplimiento, de las necesidades de la ciudadanía y su expectativa de dar valor y apoyo a la gestión de cada entidad.

En la empresa, las áreas consideradas como principales funciones financieras son las siguientes:

- Análisis de los aspectos financieros de todas las decisiones.

- La cantidad de inversión que se requerirá para generar las ventas que la empresa espera realizar.

- La forma de obtener los fondos y de proporcionar el financiamiento de los activos que requiere la empresa para elaborar los productos y servicios cuyas ventas generarán ingresos.

\section{Metodología}

El método implementado en este estudio parte de la investigación teórica, se pretende recolectar datos de la bibliografía especializada, con la finalidad de desarrollar un primer acercamiento hacia el tema objeto de estudio, para analizar a la Administración Financiera en el sector público, para poder identificar las características de los administradores financieros los cuales se encuentran involucrados en la toma de decisiones, a largo plazo. Se utiliza la investigación científica definida como una estrategia donde se observa y reflexiona sistemáticamente sobre realidades teóricas y empíricas sobre el análisis de la Administración Financiera en el sector público.

\section{Discusión}

Las finanzas generan procesos sociales 
profundamente marcados por la civilización donde requieren una estimación cuidadosa de sus fuerzas motrices y un conocimiento profundo de sus interrelaciones con la sociedad (Núñez Jover, 2007). Es el conjunto de elementos que debidamente ordenados y coordinados entre sí, persiguen la consecución de un fin común, la transparente administración de los fondos públicos.

Entre los subsistemas más importantes podemos citar: Presupuesto, Contabilidad Gubernamental, Tesorería, Nómina, Control Físico de Bienes, Deuda Pública y Convenios, los que están regidos por principios y normas técnicas destinados a posibilitar la asignación y utilización eficiente de los recursos públicos, de la cual se generen los adecuados registros que hagan efectivos los propósitos de transparencia y rendición de cuentas, es por eso que se debe tomar en cuenta los siguientes objetivos de la Administración Financiera:

\section{Tabla 1}

Resumen de las áreas temáticas, categorías, subcategorias y dimensiones
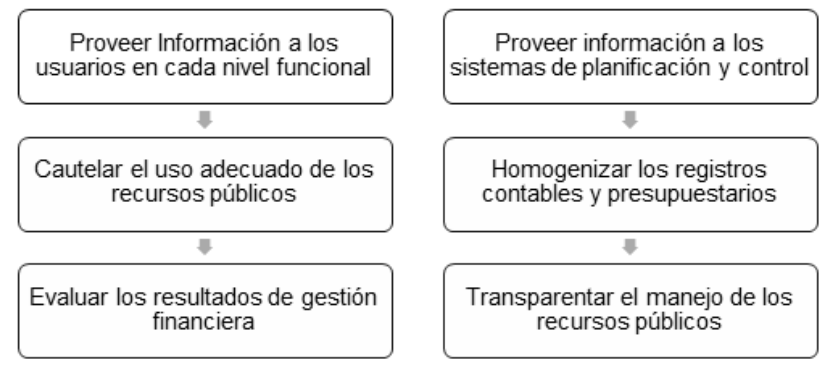

La Administración Financiera de manera directa $y$ en forma detallada debe estar presente, menciona Fred Weston (1995), con los administradores financieros ya que se encuentran involucrados con estas decisiones y deben interactuar con ellas. Se deben articular los objetivos de la administración financiera en cada uno de los procesos del sector público para evaluar las transacciones financieras, así como de la maximización del valor de la empresa en el mercado y los riesgos en las decisiones empresariales.

Según Vizcaya (2017), empezar una empresa no es fácil, la vida del emprendedor trae cada día nuevos retos y también nuevas oportunidades; sin embargo, todo es más fácil cuando se aprovechan las oportunidades que se presentan para promocionar la empresa, con el propósito de atraer más clientes y tener mayores posibilidades de expansión.

\section{Presupuesto}

Se registran tanto los ingresos que se esperan obtener, así como los diferentes gastos en que se incurrirán en base al financiamiento previsto. En otras palabras, por un lado, refleja el origen de las fuentes de financiamiento, las cuales se obtienen a través de las diversas fuentes tributarias y no tributarias y, por otro lado, el destino que se le dará a los recursos financieros durante su vigencia.

Las normas técnicas presupuestarias rigen para todas las instituciones del sector público no financiero, es decir para aquellas que no manejan efectivo de manera directa, cuya aplicación es de carácter obligatorio, pues no se pueden administrar recursos financieros en forma extrapresupuestaria, además el presupuesto debe mostrar un equilibrio entre ambos rubros, los ingresos deben ser siempre iguales a los gastos.

Las máximas autoridades de cada entidad deben remitir las proformas institucionales anuales y la programación cuatrianual de ingresos, egresos permanentes y no permanentes, en el plazo que el ente rector de las finanzas establezca. En caso de existir observaciones por parte de la Asamblea Nacional, la Función Ejecutiva tiene un plazo de diez días para remitir una nueva propuesta. La Asamblea Nacional por su parte, tiene diez días más para ratificar sus observaciones en un solo debate, caso contrario, se darán por aprobadas.

Los presupuestos de estas entidades constan de las siguientes partes: ingresos, egresos y disposiciones generales, además de un anexo con el detalle distributivo de los sueldos y salarios, el cual debe ser preparado antes del 10 de septiembre de cada año. La máxima autoridad ejecutiva del gobierno autónomo descentralizado debe presentar al órgano legislativo local el presupuesto final hasta el 31 de 
octubre, el cual va acompañado de los informes y documentos que explican los cambios realizados en las provisiones de los ingresos y gastos, así como la liquidación del presupuesto del año anterior y un estado de ingresos y gastos efectuados durante el primer semestre del año en curso.

No se aprobará el presupuesto de los GAD si estos no destinan como mínimo el $10 \%$ de sus ingresos no tributarios a la planificación o creación de programas sociales que brinden asistencia a grupos vulnerables (COOTAD, 2010). Se prohíbe que las instituciones tanto públicas como privadas otorguen créditos a los GAD que excedan estos límites de endeudamiento (Código Orgánico de Planificación y Finanzas Públicas, 2010).

La Constitución de la República del Ecuador (artículos 294 y 295) en su función ejecutiva, elabora cada año la proforma presupuestaria anual y la programación presupuestaria cuatrianual las cuales serán remitidas a la Asamblea Nacional para aprobación durante los primeros noventa días de su gestión y en los años siguientes sesenta días antes del inicio del año fiscal respectivo.

El Código Orgánico de Planificación y Finanzas Públicas, COPLAFIP (artículo 5, numeral 1) entre otras disposiciones, estipula que en todas las fases del ciclo presupuestario los presupuestos públicos y todos los recursos públicos se sujetarán a los lineamientos de la planificación del desarrollo, de igual manera el artículo 100 del COPLAFIP, dispone que las proformas de las instituciones que conforman el Presupuesto General del Estado deben elaborarse de conformidad con el Plan Nacional de Desarrollo, la programación fiscal y las directrices presupuestarias.

Entre las atribuciones del Ministerio de Economía y Finanzas, como ente rector del Sistema Nacional de Finanzas Públicas, SINFIP, señaladas en el COPLAFIP (artículos 74, numeral 6 y 101), se establece dictar las directrices para el diseño, implementación y cumplimiento del SINFIP, las mismas que son de cumplimiento obligatorio por parte de las entidades del sector público, incluidas las empresas públicas, gobiernos autónomos descentralizados, banca pública y seguridad social.

La Secretaría Nacional de Planificación y Desarrollo, como ente rector de la inversión pública, tiene la competencia de formular los planes de inversión del Presupuesto General del Estado, las entidades que lo conforman deben presentar los proyectos de inversión, para la obtención del dictamen de prioridad, con el fin de ser incluidos en el Plan Anual y Plurianual de Inversiones, y su correspondiente financiamiento.

\section{Código Orgánico de Planificación y Finanzas Públicas}

Todas las entidades públicas engloban un conjunto de organismos, recursos, sistemas y procedimientos que debidamente ordenados y coordinados entre si buscaban trasparentar el manejo de los fondos económicos que poseen las organizaciones, es decir, la captación y utilización eficiente de los recursos públicos, con el fin de concretar las metas propuestas por el Estado, las mismas que se dan a conocer a través de las rendiciones de cuentas periódicas que deben realizar (Ley Orgánica de Administración Financiera y Control, 2001).

Sin embargo, en el campo de las finanzas públicas existían varios cuerpos legales, que debido a su dispersión causaba que su aplicación se torne confusa y algunas veces hasta contradictoria.

Es por esto que para el 2010 se realizó una reforma más profunda a la legislación sobre finanzas, con el objetivo de que los programas económicos de ahora en adelante también incluyan garantías del buen vivir, participación ciudadana y estabilidad fiscal a mediano y largo plazo.

Todos los organismos son dirigidos por el Gobierno Central los cuales forman parte de un solo Ente Financiero, que de acuerdo al Ministerio de Economía y Finanzas se manejan por medio de una contabilidad centralizada y un presupuesto desconcentrado, los mismos que son administrados por medio de la caja fiscal (Normativa del Sistema de Administración Financiera, pág. 4). 
El COOTAD (Código Orgánico de Organización Territorial), se encarga de establecer la organización administrativa del Estado en los diferentes niveles de GAD (Gobierno Autónomo Descentralizado) y regímenes especiales, este código desarrolla un modelo de descentralización obligatorio para todos, el cual busca reparar los desequilibrios existentes en el desarrollo territorial (COOTAD, 2010).

\section{Resultados}

Las Finanzas se encargan de entender las causas y consecuencias de las transacciones financieras, así como de la maximización del valor de la empresa en el mercado y de la medida de los riesgos en las decisiones empresariales, es un ejemplo vivo de aciertos previos. Es preciso señalar dentro de la función la contabilidad como ciencia económica, se encarga de entender su devenir histórico por lo que propone dirigir la discusión en términos de las ventajas y desventajas de implementar presupuestos dirigidos a resultados, partiendo de la premisa de que las Instituciones dependiendo del presupuesto no necesariamente van a cambiar la presupuestación sabiendo que es una actividad técnica en operación, pero política en naturaleza.

Para Brugué y Goma (1998), la política pública incluye mecanismos de asignación de recursos y oportunidades, entre diferentes grupos sociales, como se observa existen una serie de elementos que se conjugan para que las Finanzas y las Políticas Públicas sean compatibles entre sí, en el caso de estas últimas, pasen por un proceso de validación social en el que el ciudadano especifique las demandas sociales que deben tener respuesta por parte del Estado, mediante las metas establecidas en sus instituciones.

Según Ayuda Urbana (2002), el manejo de las finanzas locales permite erradicar muchos de los problemas que afectan el desarrollo y bienestar de las comunidades en la esfera empresarial, el desarrollo de las finanzas implica la ocurrencia de cambios en la administración financiera, en un mundo que se vinculó estrechamente con los requerimientos del registro y análisis sistemático de las operaciones derivadas de la complejidad de las actividades comerciales, el surgimiento del proceso de industrialización y la internacionalización de las empresas.

El análisis del impacto que tienen las finanzas en la economía y en la sociedad no puede realizarse al margen del papel que juegan en ello la ciencia y la tecnología ya que, como procesos sociales profundamente marcados por la civilización donde han crecido, requieren una estimación cuidadosa de sus fuerzas motrices e impactos y un conocimiento profundo de sus interrelaciones con la sociedad (Núñez, 2007)

\section{Conclusiones}

La participación de los ciudadanos en las diferentes fases del proceso de formulación de políticas públicas es, en principio, deseable y conveniente. Como regla general, esta parece bastante razonable. No obstante, puede demostrarse que incluso esta regla de inspiración democrática no es aplicable a todos los niveles de la acción pública. De un lado tenemos la participación efectiva, con igualdad de oportunidades para los diferentes sectores interesados, donde a nivel local es más fácil incentivar a sectores afectados, aunque inicialmente menos motivados y poco organizados, sin debilitar demasiado la capacidad decisoria del Estado.

En cambio, a nivel nacional, la participación se ve más restringida a sectores altamente organizados y con gran capacidad de movilización. El Sistema de Administración Financiera para la gestión del Sector Público como un conjunto de elementos interrelacionado que persiguen la consecución de una administración transparente de los recursos públicos; la normativa vigente en el Ecuador garantiza, dispone, es decir, obliga el cumplimiento de una gestión eficiente, eficaz y calidad de los servicios a la comunidad.

El integrar: Presupuesto, Contabilidad Gubernamental, Tesorería, Nómina, Control Físico de Bienes, Deuda Pública y Convenios, permite alcanzar los objetivos y metas trazadas por las diferentes instituciones del Estado causando un impacto en las áreas: Educación, Salud, Empleo, 
Vivienda, Desigualdad y pobreza, Inversión social, Desarrollo infantil, Acción social, Población y Economía.

\section{Referencias bibliográficas}

Ayuda Urbana, Red Intermunicipal. (2002). Finanzas Municipales ¿Qué es? www. ayudaurbana.com/pages/finanzas/que_es.

Brugue, Q. y Goma, R. (1998), Gobiernos locales y políticas públicas. Bienestar social, promoción económica y territorio, Barcelona, Editorial Ariel S.A

Cardoso, G., Bermeo, E., y Fresa, M. (2012). Ecuador: Economía y Finanzas Populares y Solidarias, para el Buen Vivir Quito: Editorial Imprefepp.

Código Orgánico de Finanzas Públicas y Planificación.(2010).https://www.finanzas.gob. ec/wp-content/uploads/downloads/2012/09/ CODIGO_PLANIFICACION_FINAZAS.pdf

Código Orgánico de Ordenamiento Territorial Autonomía y Descentralización. (2010). https:// www.oas.org/juridico/pdfs/mesicic4_ecu_org. pdf

Constitución de la República del Ecuador. (2008). https://www.wipo.int/edocs/lexdocs/laws/es/ ec/ec030es.pdf

Constancio, R. (1996). Un Modelo para Interpretar los Vínculos entre Presupuesto Público y Estabilidad Política. Política y Cultura 7: 289302.

Franciskovic, J. (2013). Retos de la Gestión Pública: Presupuesto por Resultados y Rendición de Cuentas. Journal of Economics, Finance and Administrative Science 18: 28-32.

Gómez, C. (2004). El Presupuesto Público En la Gestión Eficiente de los Municipios. INNOVAR. Revista de Ciencias Administrativas y Sociales 24: $105-11$.
Gutiérrez, A. (2015). Gasto Público y Presupuesto Base Cero En Mexico. El Cotidiano 192: 13-32.

Hernández, J. (2014). Reinventando la Política Fiscal: ¿Una Nueva Estrategia para la Estabilización y el Crecimiento Económico? Cuadernos de Economía 2: 33-60.

Manual de Contabilidad Gubernamental. (2010). https://www.finanzas.gob.ec/wp-content/ uploads/downloads/2012/09/Manualcontabilidad-CAPITULOIYII.pdf

Núñez, J. (2007): La ciencia y la tecnología como procesos sociales. Lo que la educación científica no debería olvidar, Félix Varela, La Habana.

Normas Técnicas de Presupuesto. (2011). https:// www.finanzas.gob.ec/wp-content/uploads/ downloads/2012/09/Normativa-PresupuestariaActualizada-al-11-10-2011.pdf

Pinilla, D.; Jiménez, J.; Montero, R. (2013). "Gasto Público y Crecimiento Económico: Un Estudio Empírico en América Latina. Cuadernos de Economía 32: 181-210.

Vizcaya, P. (2017). La papelería corporativa de un emprendedor. https://www.printu.co/blog/ identidad-corporativa/papeleriacorporativa

Weston, J. F., y Thomas, C. (1995). Finanzas en Administración. Novena edición. Vol. I y II. Mc GrawHill. 